\section{Acesso e qualidade da atenção pré-natal na Estratégia Saúde da Família: infraestrutura, cuidado e gestão}

\author{
Access to prenatal care and quality of care in the \\ Family Health Strategy: infrastructure, care, \\ and management
}

\section{Acceso y calidad de la atención prenatal en la Estrategia Salud de la Familia: infraestructura, cuidado y gestión}

\section{Resumo}

Investigação do acesso e da qualidade do cuidado pré-natal na Estratégia Saúde da Família no Brasil e na Região Norte, mediante avaliação de aspectos de infraestrutura nas unidades de saúde, da gestão e oferta do cuidado prestado pelas equipes, sob o prisma das desigualdades regionais e estaduais. Foi realizado um estudo transversal, avaliativo de tipo normativo, derivado do componente de avaliação externa do segundo ciclo do Programa de Melhoria do Acesso e da Qualidade da Atenção Básica, realizado entre 2013-2014. Os resultados evidenciam inadequação da infraestrutura da rede de atenção básica que realiza o pré-natal; baixa adequação de ações clínicas para a qualidade do cuidado e baixa capacidade de gestão das equipes para garantir o acesso e qualidade do cuidado. Na distribuição por regiões geopoliticas, os achados relativos à infraestrutura das unidades apontam uma relação direta entre adequação da infraestrutura e contextos sociais com Índice de Desenvolvimento Humano Municipal e renda mais elevados. Para as ações clinicas do cuidado, as equipes de todas as regiões obtiveram indices baixos de adequação, tendo sido observado resultados discretamente mais elevados nas regiões Norte e Sul. Houve diferenças expressivas entre os estados da Região Norte, obtendo melhor adequação as unidades federadas com melhores condições de renda e de desenvolvimento humano. Os resultados indicam importantes dificuldades organizacionais tanto no acesso, quanto na qualidade do cuidado ofertado pelas equipes de saúde, além de uma evidente insuficiência das ações de gestão voltadas ao aprimoramento do acesso e da qualidade do cuidado pré-natal.

Atenção Primária à Saúde; Cuidado Pré-Natal; Avaliação em Saúde; Desigualdades em Saúde
Wilderi Sidney Gonçalves Guimarães 1 Rosana Cristina Pereira Parente 2

Thayanne Louzada Ferreira Guimarães 1 Luiza Garnelo 2

doi: 10.1590/0102-311X00110417
Correspondência

W. S. G. Guimarães

Departamento de Saúde Coletiva, Faculdade de Medicina, Universidade Federal do Amazonas.

Rua Afonso Pena 1053, Manaus, AM 69020-160, Brasil.

wilderi.sidney@gmail.com

1 Faculdade de Medicina, Universidade Federal do Amazonas, Manaus, Brasil.

2 Instituto Leônidas e Maria Deane, Fundação Oswaldo Cruz, Manaus, Brasil. 


\section{Introdução}

A atenção pré-natal visa contribuir para a redução da morbimortalidade materna e infantil 1,2. Um pré-natal de qualidade deve desenvolver ações resolutivas e acolhedoras para as gestantes na rede de atenção básica, bem como coordenar e facilitar o acesso oportuno à própria atenção básica e a outros níveis de atenção da rede de serviços de saúde, buscando garantir a oferta adequada de cuidados com a gestação e o parto ${ }^{3}$.

O conhecimento das ações de pré-natal é relevante para o entendimento dos principais avanços alcançados e dos obstáculos a serem superados pelo Sistema Único de Saúde (SUS) na melhoria dos indicadores de saúde materna. A atuação da Estratégia Saúde da Família (ESF), principal modelo de atenção para a efetivação do pré-natal na rede básica do SUS, tem sido alvo de investigações sobre o tema 1,2,4,5. Dentre os desafios à melhoria da qualidade da atenção pré-natal, aponta-se a necessidade de institucionalizar o monitoramento e avaliação da rotina de suas ações, implementadas pelas equipes de saúde da família (EqSF).

Dentre os esforços para fortalecer a avaliação em saúde no Brasil, destaca-se o Programa Nacional de Melhoria do Acesso e da Qualidade na Atenção Básica (PMAQ-AB) que, além da preocupação em avaliar globalmente o desempenho da atenção básica, também gerou um amplo conjunto de informações sobre o pré-natal 6.

O pré-natal tem sido objeto de grande número de estudos 4,7,8 com destaque para avaliação da adequação das suas ações, guiada pelos critérios de qualidade e efetividade estabelecidos pelo Programa de Humanização do Pré-natal e Nascimento (PHPN) 1. Boa parte da literatura disponível aponta baixa qualidade no cuidado de pré-natal ofertado na atenção básica 1,2,4 e a persistência de desigualdades regionais e sociodemográficas no acesso, resolutividade e desfecho da atenção pré-natal 2,8,9,10,11,12. Soma-se a isso, a carência de estudos avaliativos do processo de gestão do acesso e do cuidado ofertado pelas EqSF em relação ao pré-natal.

No que diz respeito às regiões geográficas brasileiras, a literatura tem constatado uma confluência de menor adequação, menor cobertura e o pior desempenho na Região Norte 7,8,10. Nessa região também são elevados os níveis de pobreza, há falta de pessoal de saúde e limitada cobertura assistencial. São características que atestam a persistência de acentuadas desigualdades sociais e sanitárias no território nacional 2,8.

Sob o eixo das desigualdades regionais o artigo investiga acesso e qualidade do cuidado pré-natal por meio de avaliação de aspectos de infraestrutura nas unidades de saúde da família (USF), das ações gerenciais que favoreçam o acesso e potencializem a qualidade dos cuidados prestados pelas EqSF, no Brasil e na Região Norte.

\section{Metodologia}

Estudo transversal, avaliativo de tipo normativo, derivado do componente de avaliação externa do segundo ciclo do PMAQ-AB.

O instrumento de avaliação externa dispunha de três módulos que orientaram a coleta de dados: Módulo I - observação na USF; Módulo II - entrevista com um profissional sobre o processo de trabalho da EqSF e verificação documental; Módulo III - entrevista com usuário na unidade de saúde 13,14. Neste estudo foram utilizadas variáveis contidas nos Módulos I e II.

O banco resultante do segundo ciclo de avaliação externa traz informações coletadas entre 2013 e 2014, junto às equipes que aderiram voluntariamente ao programa. Ele disponibilizou dados relativos a 24.055 USF brasileiras e às entrevistas com profissionais de 29.778 EqSF no Brasil, o equivalente a $86 \%$ das equipes atuantes no território nacional à época da coleta, de acordo com dados do site do Departamento de Atenção Básica do Ministério da Saúde (http://dab.saude.gov.br/portaldab/ historico_cobertura_sf.php).

O manual técnico do pré-natal 3 orientou a escolha de questões no banco de dados do PMAQ-AB, priorizando-se as que permitiam avaliar o acesso e a qualidade do cuidado pré-natal 15,16.

$\mathrm{O}$ acesso, avaliado por intermédio da relação entre os diversos elementos técnicos que caracterizam a oferta da atenção pré-natal, foi entendido como a facilidade ou dificuldade com que as pessoas 
obtêm tais cuidados de saúde 15 , investigando-se as barreiras impostas às gestantes pelos próprios serviços de pré-natal. Essa estratégia avaliativa prioriza dimensões da oferta dos serviços de saúde, sendo empreendida pelas seguintes subdimensões: disponibilidade de infraestrutura das USF (dados obtidos no Módulo I do instrumento de avaliação externa) e das ações gerenciais das EqSF (dados obtidos no Módulo II do instrumento da avaliação externa), voltadas para garantir o acesso ao cuidado.

A avaliação da qualidade do cuidado diz respeito ao conjunto de atributos do processo de trabalho, levando-se em conta o conhecimento, as tecnologias e as normas sociais vigentes, segundo aspectos técnicos, interpessoais e organizacionais 16 .

Neste artigo, a avaliação da qualidade do cuidado da atenção pré-natal priorizou a análise organizacional dos serviços do pré-natal, nas subdimensões ações gerenciais para garantir a qualidade do cuidado e cuidados clínicos ofertados (ambas mediante os dados obtidos no Módulo II do instrumento da avaliação externa).

Foi construída uma matriz de julgamento 17 para auxiliar na análise e interpretação das informações relativas às categorias (dimensões), critérios (subdimensões), indicadores de adequação e parâmetros de referência (questões do PMAQ-AB relativas ao pré-natal). As dimensões e subdimensões de análise estão sistematizadas na Tabela 1.

As equipes avaliadas foram classificadas em três categorias, de acordo com o desempenho alcançado: quando todos os itens avaliados alcançaram $100 \%$ de respostas positivas, o desempenho da equipe foi considerado "adequado"; alcançando entre $80,1 \%$ a 99,9\% de positividade, o desempenho foi considerado "parcialmente adequado" e, se menor ou igual que $80 \%$ para os itens avaliados, foi classificado como "inadequado". O escalonamento também está apresentado na Tabela 1. O procedimento tomou como base os parâmetros desenvolvidos em outro estudo 18 que avaliou ação programática na ESF, utilizando igualmente o banco de dados do componente de avaliação externa do PMAQ-AB.

Para as questões conjugadas, foram avaliadas como positivas apenas as respostas que comprovassem, com documentação, a afirmação feita pelo entrevistado. As demais foram pontuadas tão somente com a resposta afirmativa do entrevistado.

Procedeu-se em seguida à classificação da adequação para as subdimensões analisadas (disponibilidade de infraestrutura, ações gerenciais para garantia do acesso, ações gerenciais para a qualidade do cuidado e ações clínicas) e as seguintes características: Brasil e suas macrorregiões geopolíticas (Norte, Nordeste, Centro-oeste, Sudeste e Sul) e os estados da Região Norte (Acre, Amapá, Amazonas, Pará, Rondônia, Roraima e Tocantins).

Foi cruzada a classificação de adequação da atenção pré-natal para a subdimensão disponibilidade de infraestrutura das USF com os valores, por contexto geográfico, do Índice de Desenvolvimento Humano Municipal (IDH-M), da cobertura populacional pela atenção básica e da renda domiciliar per capita mensal, retirados do banco de indicadores do site Região e Redes (http://www.resbr.net. br/indicadores/view/) e do site do Programa das Nações Unidas para o Desenvolvimento (PNUD; http://www.br.undp.org/content/brazil/pt/home/library/idh1/macro-regioes-brasileiras.html) no Brasil. E, por fim, foi analisada a classificação de adequação do pré-natal nos sete estados da Região Norte com os mesmos indicadores citados acima, tendo sido acrescida a taxa de mortalidade neonatal precoce para a última análise.

Os procedimentos de análise foram descritivos e analíticos, demonstrando-se as frequências e percentuais para a apresentação das ações de pré-natal por regiões geográficas. Para a verificação da associação entre adequação da atenção pré-natal nas subdimensões avaliadas e as regiões geográficas, foi feita análise bivariada pelo teste qui-quadrado ou qui-quadrado da máxima verossimilhança, quando a pressuposição do teste era atendida, no nível de significância de $5 \%$.

Para a apresentação das variáveis cobertura da atenção básica e renda mensal total domiciliar per capita, calculou-se a mediana; para o IDH-M, utilizou-se o valor por região e por estado. Os softwares utilizados foram o Excel 2013 (Microsoft Corp., Estados Unidos) e o IBM SPSS, versão 22 (IBM Corp., Armonk, Estados Unidos).

A justificativa do agrupamento em estratos, por contexto geográfico, baseia-se na diretriz do próprio $\mathrm{PMAQ}-\mathrm{AB}$, que propõe a construção de parâmetros de comparação para analisar o acesso e a qualidade dos serviços de atenção básica em nível local, regional e nacional, aferindo as possíveis desigualdades em saúde nos diferentes contextos brasileiros 13,14 . O detalhamento da análise para a 
Tabela 1

Matriz de julgamento com categorias de avaliação do acesso e da qualidade do cuidado da atenção pré-natal na Estratégia Saúde da Família.

\begin{tabular}{|c|c|c|c|}
\hline Dimensão & Subdimensão & Indicador de adequação & $\begin{array}{l}\text { Variáveis selecionadas do instrumento de coleta de dados } \\
\text { do PMAQ-AB para avaliar o pré-natal }\end{array}$ \\
\hline Acesso & $\begin{array}{l}\text { Disponibilidade de } \\
\text { infraestrutura } \\
\text { (19 questões) }\end{array}$ & $\begin{array}{c}\text { (a) de } 0 \text { a } 80 \% \text { resposta } \\
\text { positiva para até } 15 \text { itens } \\
\text { (b) } 80,1 \% \text { a } 99,9 \% \\
\text { resposta positiva para } 16 \\
\text { a } 18 \text { itens } \\
\text { (c) } 100 \% \text { resposta positiva } \\
\text { em todos os itens } \\
\text { avaliados }\end{array}$ & $\begin{array}{l}\text { - Médico na equipe } \\
\text { - Esfigmomanômetro adulto } \\
\text { - Balança antropométrica de 150kg } \\
\text { - Régua antropométrica } \\
\text { - Estetoscópio adulto } \\
\text { - Glicosímetro } \\
\text { - Mesa ginecológica com perneira } \\
\text { - Sonar } \\
\text { - Termômetro clínico } \\
\text { - Fita métrica } \\
\text { - Espéculo descartável } \\
\text { - Tiras reagentes de glicemia capilar } \\
\text { - Caderneta da gestante impressa na USF } \\
\text { - Teste rápido para sífilis } \\
\text { - Teste rápido gravidez } \\
\text { - Teste rápido para HIV } \\
\text { - Oferta regular de vacinação } \\
\text { - Imunização para tétano } \\
\text { - Imunização para hepatite B }\end{array}$ \\
\hline & $\begin{array}{c}\text { Ações gerenciais para } \\
\text { garantia do acesso } \\
\text { (11 questões) }\end{array}$ & $\begin{array}{l}\text { (a) de } 0 \text { a } 80 \% \text { resposta } \\
\text { positiva para até } 7 \text { itens } \\
\text { (b) } 80,1 \% \text { a } 99,9 \% \\
\text { resposta positiva para } 8 \\
\text { a } 9 \text { itens } \\
\text { (c) } 100 \% \text { resposta positiva } \\
\text { em todos os itens } \\
\text { avaliados }\end{array}$ & $\begin{array}{l}\text { - Realização de acolhimento à demanda espontânea } \\
\text { - Existência de protocolos para atendimento à demanda } \\
\text { espontânea } \\
\text { - Registro de gestantes de risco encaminhadas para outros } \\
\text { pontos de atenção da rede * } \\
\text { - Programação da agenda de acordo com o risco classificado * } \\
\text { - Agendamento presencial } \\
\text { - Agendamento por telefone } \\
\text { - Agendamento pela Internet } \\
\text { - Registro de todas as gestantes do território * } \\
\text { - Programação de oferta de consultas * } \\
\text { - Uso de protocolos para estratificação de risco * } \\
\text { - Realização de busca ativa * } \\
\text { - Agendamento por fila para pegar senhas }\end{array}$ \\
\hline $\begin{array}{l}\text { Qualidade do } \\
\text { cuidado }\end{array}$ & $\begin{array}{c}\text { Ações gerenciais para } \\
\text { garantia da qualidade } \\
\text { do cuidado } \\
\text { (9 questões) }\end{array}$ & $\begin{array}{l}\text { (a) de } 0 \text { a } 80 \% \text { resposta } \\
\text { positiva para até } 6 \text { itens } \\
\text { (b) } 80,1 \% \text { a } 99,9 \% \\
\text { resposta positiva para } 7 \\
\text { itens } \\
\text { (c) } 100 \% \text { resposta positiva } \\
\text { em todos os itens } \\
\text { avaliados }\end{array}$ & $\begin{array}{l}\text { - Uso da caderneta ou cartão para o acompanhamento } \\
\text { - Registro sobre consulta odontológica } \\
\text { - Registro sobre atualização vacinal } \\
\text { - Registro sobre a coleta de colpocitologia oncótica } \\
\text { - Registro de peso e altura em prontuário, cadernetas, sistema } \\
\text { de informação ou outros } \\
\text { - Alimentação mensal do sistema de informação } \\
\text { - Monitoramento pós-parto através de contra referência } \\
\text { da maternidade } \\
\text { - Monitoramento pós-parto por ações inespecíficas }\end{array}$ \\
\hline
\end{tabular}

(continua) 
Matriz de julgamento com categorias de avaliação do acesso e da qualidade do cuidado da atenção pré-natal na Estratégia Saúde da Família.

\begin{tabular}{|c|c|c|c|}
\hline Dimensão & Subdimensão & Indicador de adequação & $\begin{array}{l}\text { Variáveis selecionadas do instrumento de coleta de dados } \\
\text { do PMAQ-AB para avaliar o pré-natal }\end{array}$ \\
\hline & $\begin{array}{l}\text { Ações clínicas } \\
\text { (17 questões) }\end{array}$ & $\begin{array}{l}\text { (a) de } 0 \text { a } 80 \% \text { resposta } \\
\text { positiva para até } 11 \text { itens } \\
\text { (b) } 80,1 \% \text { a } 99,9 \% \\
\text { resposta positiva para } 12 \\
\text { a } 15 \text { itens } \\
\text { (c) } 100 \% \text { resposta positiva } \\
\text { em todos os itens } \\
\text { avaliados }\end{array}$ & $\begin{array}{l}\text { - Solicitação e realização dos seguintes exames pela rede de } \\
\text { atenção: hematócrito, glicemia de jejum, VDRL, sorologias para } \\
\text { HIV, hepatite B, toxoplasmose, testes rápidos para gravidez, } \\
\text { sífilis, HIV e urina tipo I } \\
\text { • Recepção, pela equipe, de todos os exames em } \\
\text { tempo oportuno } \\
\text { - Realização de aplicação da penicilina g benzatina na } \\
\text { própria USF } \\
\text { - Realização de consulta de puerpério até } 10 \text { dias após o parto } \\
\text { - Consulta puerperal, em domicílio, pelo ACS } \\
\text { - Consulta puerperal, em domicílio, por outros membros } \\
\text { da equipe } \\
\text { - Realização/Coleta de exames de colpocitologia na própria USF } \\
\text { - Oferta de ações educativas e de promoção da saúde }\end{array}$ \\
\hline
\end{tabular}

ACS: agente comunitário de saúde; PMAQ-AB: Programa Nacional de Melhoria do Acesso e da Qualidade na Atenção Básica; USF: unidades de saúde da família.

Fonte: elaborado pelos autores, com base no instrumento de coleta de dados da avaliação externa do PMAQ-AB, manual técnico sobre pré-natal e puerpério 3, Travassos \& Martins 15 e Champagne et al. ${ }^{16}$.

* Questões conjugadas são as que exigiam comprovação documental da ação relatada pelo entrevistado.

Região Norte se justifica pelos achados da pouca literatura que tratou do assunto, sugerindo comprometimento da qualidade da atenção pré-natal no norte do país.

A pesquisa foi aprovada em Comitê de Ética em Pesquisa da Universidade Federal do Amazonas (processo no 1.385.179/2016).

\section{Resultados}

A Tabela 2 apresenta os resultados da adequação do pré-natal relativos ao acesso e qualidade do cuidado encontrado para o Brasil e para as cinco macrorregiões geopolíticas brasileiras.

Para a dimensão acesso, a atenção pré-natal foi considerada inadequada no Brasil como um todo, tanto na subdimensão da disponibilidade de infraestrutura das USF (26\% das equipes com infraestrutura para atenção pré-natal adequada e 31\% com adequação parcial), quanto na subdimensão das ações gerenciais para garantia do acesso (11\% adequadas e $27 \%$ parcialmente adequadas).

Na distribuição por regiões, a disponibilidade de infraestrutura teve os piores resultados nas USF do Norte (18\% de adequação e 31\% das unidades parcialmente adequadas) e do Centro-oeste (22\% adequadas e $30 \%$ de adequação parcial). Foi nas regiões Nordeste (29\% das unidades de saúde com infraestrutura adequada e 26\% com adequação parcial) e Sul (26\% adequação e 43\% parcialmente adequadas para oferta do pré-natal) que as unidades de saúde alcançaram os melhores índices de adequação para a subdimensão do acesso.

Na subdimensão das ações gerenciais para garantia do acesso, os destaques negativos foram nas regiões Nordeste (4\% adequação e $28 \%$ adequação parcial), Centro-oeste (5\% adequação e $23 \%$ adequação parcial e Norte (5\% de adequação e $21 \%$ de parcialmente adequadas). Por outro lado, as regiões Sudeste (21\% adequadas e 30\% adequação parcial) e Sul (14\% adequação e 27\% parcialmente adequadas) obtiveram melhores desempenhos em tal atributo.

A dimensão da qualidade do cuidado pré-natal foi avaliada como nacionalmente inadequada. A análise da subdimensão ações gerenciais evidencia restrito nível de adequação (5\% adequação e 29\% 
Tabela 2

Distribuição da adequação da atenção pré-natal relativa ao acesso e qualidade do cuidado no Brasil e suas cinco macrorregiões geopolíticas, 2014.

\begin{tabular}{|c|c|c|c|c|c|c|c|c|c|}
\hline Dimensões & Subdimensões & Intervalos & Brasil & Norte & Nordeste & Centro-oeste & Sudeste & Sul & Valor de $p$ \\
\hline \multirow[t]{12}{*}{ Acesso } & Disponibilidade de & $0 \%$ a $80 \%$ & 10.291 & 870 & 4.296 & 900 & 3.089 & 1.136 & \multirow{6}{*}{$<0,001$} \\
\hline & infraestrutura & & $43 \%$ & $51 \%$ & $44 \%$ & $48 \%$ & $43 \%$ & $31 \%$ & \\
\hline & & \multirow[t]{2}{*}{$80,1 \%$ a $99 \%$} & 7.459 & 520 & 2.558 & 573 & 2.262 & 1.546 & \\
\hline & & & $31 \%$ & $31 \%$ & $26 \%$ & $30 \%$ & $32 \%$ & $43 \%$ & \\
\hline & & \multirow[t]{2}{*}{$100 \%$} & 6.305 & 300 & 2.850 & 416 & 1.814 & 925 & \\
\hline & & & $26 \%$ & $18 \%$ & $29 \%$ & $22 \%$ & $25 \%$ & $26 \%$ & \\
\hline & Ações gerenciais & $0 \%$ a $80 \%$ & 18.187 & 1.600 & 7.355 & 1.600 & 4.969 & 2.663 & \multirow{6}{*}{$<0,001$} \\
\hline & para garantia do & & $61 \%$ & $74 \%$ & $68 \%$ & $71 \%$ & $49 \%$ & $59 \%$ & \\
\hline & \multirow[t]{4}{*}{ acesso } & \multirow[t]{2}{*}{$80,1 \%$ a $99 \%$} & 8.196 & 450 & 3.009 & 517 & 2.995 & 1.225 & \\
\hline & & & $27 \%$ & $21 \%$ & $28 \%$ & $23 \%$ & $30 \%$ & $27 \%$ & \\
\hline & & \multirow[t]{2}{*}{$100 \%$} & 3.395 & 110 & 404 & 124 & 2.136 & 621 & \\
\hline & & & $11 \%$ & $5 \%$ & $4 \%$ & $5 \%$ & $21 \%$ & $14 \%$ & \\
\hline \multirow[t]{12}{*}{ Qualidade } & \multirow{6}{*}{$\begin{array}{l}\text { Ações gerenciais } \\
\text { para qualidade do } \\
\text { cuidado }\end{array}$} & \multirow[t]{2}{*}{$0 \%$ a $80 \%$} & 19.412 & 1.619 & 7.541 & 1.621 & 5.997 & 2.634 & \multirow{6}{*}{$<0,001$} \\
\hline & & & $65 \%$ & $75 \%$ & $70 \%$ & $72 \%$ & $59 \%$ & $58 \%$ & \\
\hline & & \multirow[t]{2}{*}{$80,1 \%$ a $99 \%$} & 8.768 & 507 & 3.023 & 540 & 3.165 & 1.533 & \\
\hline & & & $29 \%$ & $23 \%$ & $28 \%$ & $24 \%$ & $31 \%$ & $34 \%$ & \\
\hline & & \multirow[t]{2}{*}{$100 \%$} & 1.598 & 34 & 204 & 80 & 938 & 342 & \\
\hline & & & $5 \%$ & $2 \%$ & $2 \%$ & $4 \%$ & $9 \%$ & $8 \%$ & \\
\hline & \multirow{6}{*}{$\begin{array}{c}\text { Ações clínicas para } \\
\text { a qualidade do } \\
\text { cuidado }\end{array}$} & \multirow[t]{2}{*}{$0 \%$ a $80 \%$} & 18.906 & 1.293 & 7.119 & 1.447 & 6.398 & 2.649 & \multirow{6}{*}{$<0,001$} \\
\hline & & & $63 \%$ & $60 \%$ & $66 \%$ & $65 \%$ & $63 \%$ & $59 \%$ & \\
\hline & & $80,1 \%$ a $99 \%$ & 10.868 & 863 & 3.649 & 794 & 3.702 & 1.860 & \\
\hline & & & $36 \%$ & $40 \%$ & $34 \%$ & $35 \%$ & $37 \%$ & $41 \%$ & \\
\hline & & \multirow[t]{2}{*}{$100 \%$} & 4 & 4 & 0 & 0 & 0 & 0 & \\
\hline & & & $0 \%$ & $0 \%$ & $0 \%$ & $0 \%$ & $0 \%$ & $0 \%$ & \\
\hline
\end{tabular}

Fonte: avaliação externa do Programa Nacional de Melhoria do Acesso e da Qualidade na Atenção Básica (PMAQ-AB), Brasil, 2014.

parcialmente adequada). No tocante às diferenças regionais, os resultados também foram desfavoráveis para as equipes do Norte ( $2 \%$ de adequação e $23 \%$ adequação parcial), Nordeste ( $2 \%$ adequação e $28 \%$ adequação parcial) e Centro-oeste (4\% adequação e $24 \%$ adequação parcial). Equipes do Sudeste ( $9 \%$ de adequação e $31 \%$ parcialmente adequada) e Sul ( $8 \%$ adequação e $34 \%$ adequação parcial) repetiram percentuais de adequação um pouco mais elevados, ainda que, no geral, todos os resultados alcançados sejam ruins. Destaca-se o elevado percentual de inadequação entre as equipes da Região Norte (75\%).

Para o conjunto das ações clínicas, incluídas na dimensão da qualidade do cuidado, apenas 4 equipes, do total de 29.778 investigadas, informaram realizar 100\% dos itens incluídos nessa subdimensão, o que representa menos de $1 \%$ de adequação da atenção pré-natal no país. Em 36\% das equipes, a adequação encontrada foi parcial. Na distribuição por regiões, as equipes do Sul (com 41\% de adequação parcial) e Norte (40\% de parcial) alcançaram resultados discretamente mais elevados. Em contrapartida, equipes do Nordeste (34\% de adequação parcial) e Centro-oeste (35\% parcialmente adequadas) alcançaram os menores níveis de adequação nessa subdimensão do pré-natal.

O cruzamento entre adequação da atenção pré-natal, avaliada pela disponibilidade de infraestrutura (subdimensão do acesso), por regiões geográficas, indicadores socioeconômicos e de saúde (Tabela 3), mostrou que baixos índices de adequação das USF da Região Norte foram encontrados em locais com piores indicadores de renda e IDH-M. A Região Centro-oeste mostrou resultados distintos; ali coexiste a baixa adequação da atenção pré-natal com renda per capita compatível com a da Região Sudeste, bem acima da renda de Norte e Nordeste; o IDH-M do Centro-oeste é igualmente mais elevado que o existente naquelas duas regiões e tem a mais baixa cobertura assistencial 


\section{Tabela 3}

Distribuição da adequação da atenção pré-natal relativa à subdimensão disponibilidade de infraestrutura das unidades de saúde da família no Brasil de acordo com variáveis independentes.

\begin{tabular}{|c|c|c|c|c|c|c|c|c|c|}
\hline & Subdimensão & Intervalos & Brasil & Norte & Nordeste & Centro-oeste & Sudeste & Sul & Valor de $p$ \\
\hline \multirow{6}{*}{$\begin{array}{l}\text { Adequação } \\
\text { da atenção } \\
\text { pré-natal }\end{array}$} & Disponibilidade de & $0 \%$ a $80 \%$ & 10.291 & 870 & 4.296 & 900 & 3.089 & 1.136 & \multirow{6}{*}{$<0,001$} \\
\hline & infraestrutura para & & $43 \%$ & $51 \%$ & $44 \%$ & $47 \%$ & $43 \%$ & $32 \%$ & \\
\hline & o acesso & $80,1 \%$ a $99,9 \%$ & 7.459 & 520 & 2.558 & 573 & 2.262 & 1.546 & \\
\hline & & & $31 \%$ & $31 \%$ & $26 \%$ & $30 \%$ & $32 \%$ & $43 \%$ & \\
\hline & & \multirow[t]{2}{*}{$100 \%$} & 6.305 & 300 & 2.850 & 416 & 1.814 & 925 & \\
\hline & & & $26 \%$ & $18 \%$ & $29 \%$ & $22 \%$ & $25 \%$ & $26 \%$ & \\
\hline \multicolumn{3}{|c|}{$\begin{array}{l}\text { Mediana da cobertura populacional pela atenção } \\
\text { básica (2014) }\end{array}$} & 76,82 & 64,24 & 76,82 & 69,16 & 81,15 & 78,57 & \\
\hline \multicolumn{3}{|c|}{$\begin{array}{l}\text { Mediana da renda mensal total domiciliar per capita } \\
\text { em Reais (2010) }\end{array}$} & 641,00 & 457,00 & 423,00 & 685,00 & 691,00 & 810,00 & \\
\hline \multicolumn{3}{|c|}{ IDH-M (2010) } & 0,754 & 0,663 & 0,667 & 0,757 & 0,766 & 0,754 & \\
\hline
\end{tabular}

IDH-M: Índice de Desenvolvimento Humano Municipal.

Fonte: avaliação externa do Programa Nacional de Melhoria do Acesso e da Qualidade na Atenção Básica (PMAQ-AB), Brasil, 2014; sites Região e Redes (http://www.resbr.net.br/indicadores/view/) e do Programa das Nações Unidas para o Desenvolvimento (PNUD; http://www.br.undp.org/content/brazil/ pt/home/library/idh1/macro-regioes-brasileiras.html).

de atenção básica dentre todas as regiões do país (69\%). Além disso, não se constatou relação entre maior disponibilidade de infraestrutura e melhores coberturas populacionais pela atenção básica. Para a Região Sul, verificou-se o segundo maior percentual de adequação de infraestrutura, ao lado da melhor condição de renda e IDH-M.

Tendo em vista a baixa adequação da atenção pré-natal para a Região Norte, procedeu-se a uma análise mais detalhada para cada estado dessa região (zoom out), buscando aferir possíveis diferenças entre eles (Tabela 4). Para a dimensão acesso, subdimensão disponibilidade de infraestrutura, observa-se que os estados de Roraima (14\% adequação e 56\% adequação parcial) e Amapá (18\% adequação e $48 \%$ adequação parcial) obtiveram índices melhores. Os estados do Amazonas (14\% adequação e $22 \%$ parcialmente adequado) e do Pará (19\% adequação e $26 \%$ adequação parcial) tiveram desempenhos piores.

Em relação à subdimensão ações gerenciais para garantir o acesso ao pré-natal, todos os estados foram avaliados como inadequados. Com destaque para os estados do Acre (1\% adequação e 10\% parcialmente adequado) e do Amazonas (4\% adequação e 10\% parcialmente adequado).

Na dimensão qualidade do cuidado, subdimensão ações gerenciais, nota-se situação semelhante àquela evidenciada para as ações gerencias do acesso: inadequação em todos os sete estados. Entre os piores resultados, destacam-se Amazonas e Amapá com somente 1\% de adequação e 13\% de adequação parcial, para ambos.

No respeitante à subdimensão ações clínicas (dimensão qualidade do cuidado), percebe-se que Acre (67\% parcialmente adequado) e Roraima (66\% parcialmente adequado) alcançaram melhores percentagens frente aos demais estados e até mesmo em relação aos resultados encontrados para a Região Norte e para o Brasil como um todo. Por outro lado, todos os demais estados tiveram adequação abaixo de 50\%, com destaque para os estados do Pará (27\% adequação parcial), do Tocantins (43\% adequação parcial), do Amazonas e de Rondônia (43 e 44\% de adequação parcial, respectivamente).

Ainda na Tabela 4, é possível avaliar a adequação do pré-natal por estados da Região Norte, frente aos indicadores socioeconômicos e de saúde. Notam-se melhores indicadores de IDH-M e de renda domiciliar em estados que obtiveram melhores adequações da atenção pré-natal, mas Amapá e Roraima abrigam os melhores resultados de adequação e melhores índices socioeconômicos. Já Amazonas e Pará apresentam a concomitância entre piores desempenhos do pré-natal e situações de renda e IDH-M mais desfavoráveis. 


\section{Tabela 4}

Distribuição da adequação da atenção pré-natal por estados da Região Norte, Brasil, e de acordo com variáveis independentes.

\begin{tabular}{|c|c|c|c|c|c|c|c|c|c|c|c|c|c|c|c|c|}
\hline \multirow[t]{2}{*}{ Subdimensão } & \multirow{2}{*}{ Intervalos } & \multicolumn{2}{|c|}{ Acre } & \multicolumn{2}{|c|}{ Amazonas } & \multicolumn{2}{|c|}{ Amapá } & \multicolumn{2}{|c|}{ Pará } & \multicolumn{2}{|c|}{ Rondônia } & \multicolumn{2}{|c|}{ Roraima } & \multicolumn{2}{|c|}{ Tocantins } & \multirow[t]{2}{*}{ Valor de $p$} \\
\hline & & $\mathbf{n}$ & $\%$ & $\mathbf{n}$ & $\%$ & $\mathbf{n}$ & $\%$ & $\mathbf{n}$ & $\%$ & $\mathbf{n}$ & $\%$ & $\mathbf{n}$ & $\%$ & $\mathbf{n}$ & $\%$ & \\
\hline Disponibilidade & $0 \%$ a $80,0 \%$ & 35 & 38 & 237 & 64 & 23 & 34 & 369 & 55 & 77 & 45 & 16 & 30 & 116 & 44 & \\
\hline de infraestrutura & $80,1 \%$ a $99,9 \%$ & 48 & 53 & 82 & 22 & 33 & 48 & 179 & 26 & 75 & 44 & 31 & 56 & 72 & 28 & $<0,001$ \\
\hline para o acesso & $100 \%$ & 8 & 9 & 52 & 14 & 12 & 18 & 129 & 19 & 18 & 11 & 8 & 14 & 73 & 28 & \\
\hline Ações gerenciais & $0 \%$ a $80,0 \%$ & 88 & 89 & 403 & 86 & 93 & 74 & 495 & 63 & 196 & 78 & 56 & 73 & 269 & 74 & \\
\hline para garantia do & $80,1 \%$ a $99,9 \%$ & 10 & 10 & 47 & 10 & 30 & 24 & 230 & 30 & 46 & 18 & 18 & 23 & 69 & 19 & $<0,001$ \\
\hline acesso & $100 \%$ & 1 & 1 & 19 & 4 & 3 & 2 & 51 & 7 & 10 & 4 & 3 & 4 & 23 & 6 & \\
\hline Ações gerenciais & $0 \%$ a $80,0 \%$ & 74 & 75 & 404 & 86 & 109 & 86 & 532 & 68 & 176 & 69 & 44 & 57 & 280 & 78 & \\
\hline para a qualidade & $80,1 \%$ a $99,9 \%$ & 22 & 22 & 61 & 13 & 16 & 13 & 230 & 30 & 75 & 30 & 32 & 42 & 71 & 20 & $<0,001$ \\
\hline do cuidado & $100 \%$ & 3 & 3 & 4 & 1 & 1 & 1 & 14 & 2 & 1 & 0 & 1 & 1 & 10 & 3 & \\
\hline Ações clínicas & $0 \%$ a $80,0 \%$ & 33 & 33 & 264 & 56 & 61 & 48 & 564 & 72 & 141 & 56 & 26 & 33 & 204 & 57 & \\
\hline para qualidade & $80,1 \%$ a $99,9 \%$ & 66 & 67 & 201 & 43 & 65 & 52 & 212 & 27 & 111 & 44 & 51 & 66 & 157 & 43 & $<0,001$ \\
\hline do cuidado & $100 \%$ & 0 & 0 & 4 & 1 & 0 & 0 & 0 & 0 & 0 & 0 & 0 & 0 & 0 & 0 & \\
\hline \multicolumn{2}{|c|}{$\begin{array}{l}\text { Proporção de cobertura } \\
\text { populacional pela atenção básica } \\
\text { por estado (2014) }\end{array}$} & \multicolumn{2}{|c|}{91,87} & \multicolumn{2}{|c|}{64,24} & \multicolumn{2}{|c|}{88,01} & \multicolumn{2}{|c|}{53,70} & \multicolumn{2}{|c|}{75,28} & \multicolumn{2}{|c|}{78,39} & \multicolumn{2}{|c|}{90,72} & \\
\hline \multicolumn{2}{|c|}{ IDH-M por estado (2010) } & \multicolumn{2}{|c|}{0,663} & \multicolumn{2}{|c|}{0,674} & \multicolumn{2}{|c|}{0,708} & \multicolumn{2}{|c|}{0,646} & \multicolumn{2}{|c|}{0,690} & \multicolumn{2}{|c|}{0,707} & \multicolumn{2}{|c|}{0,699} & \\
\hline \multicolumn{2}{|c|}{$\begin{array}{l}\text { Renda mensal total domiciliar per } \\
\text { capita em Reais (2010) }\end{array}$} & \multicolumn{2}{|c|}{471,00} & \multicolumn{2}{|c|}{457,00} & \multicolumn{2}{|c|}{525,00} & \multicolumn{2}{|c|}{383,00} & \multicolumn{2}{|c|}{566,00} & & & 51 & & \\
\hline $\begin{array}{l}\text { Taxa de mortalidac } \\
\text { precoce (por } 1.000 \\
(2011-2013)\end{array}$ & $\begin{array}{l}\text { nascidos vivos) } \\
\text { neonatal }\end{array}$ & & & & & & & & & & & & & & & \\
\hline
\end{tabular}

IDH-M: Índice de Desenvolvimento Humano Municipal.

Fonte: avaliação externa do Programa Nacional de Melhoria do Acesso e da Qualidade na Atenção Básica (PMAQ-AB), Brasil, 2014; sites Região e Redes (http://www.resbr.net.br/indicadores/view/) e do Programa das Nações Unidas para o Desenvolvimento (PNUD; http://www.br.undp.org/content/brazil/ pt/home/library/idh1/macro-regioes-brasileiras.html).

Quanto à cobertura populacional pela atenção básica, embora não se tenha evidenciado melhor adequação da atenção pré-natal em estado com maior cobertura, foi nos estados com coberturas menores (Amazonas e Pará: 70\%) que ocorreram os piores indicadores de adequação.

\section{Discussão}

No Brasil, a maioria das pesquisas sobre pré-natal tem analisado a conformidade da organização do cuidado clínico e laboratorial ofertado às gestantes, entendida como elemento chave para o alcance de resultados favoráveis 2,4,9. Nessas abordagens, os parâmetros instituídos no PHPN se destacam, dado o grau de detalhamento das tarefas ali prescritas, o que não foi invalidado pelas normativas que o sucederam 1,5 .

As características e finalidades do PMAQ-AB permitiram efetuar um recorte mais amplo do que habitualmente encontrado na literatura sobre a atenção pré-natal, pois os achados jogaram luz, tanto sobre a infraestrutura disponível à atenção pré-natal na rede de atenção básica, quanto sobre condições de gestão que ofertam suporte necessário à melhoria do acesso e da qualidade do pré-natal. Tal singularidade é positiva por ampliar o escopo da investigação, porém dificulta a comparação com a literatura, em razão da inexistência de trabalhos de teor similar. Em função disso, a discussão será iniciada pelas subdimensões que guardam alguma equivalência com os temas já tratados na literatura, como a de infraestrutura e de ações clínicas desenvolvidas no pré-natal.

Diversos autores 19,20,21 têm avaliado a magnitude da inadequação da infraestrutura da rede básica no país, o que é corroborado pelos baixos percentuais da adequação da estrutura física das USF 
dirigida ao pré-natal, aqui encontrados para o Brasil como um todo (26\% unidades adequadas e $31 \%$ parcialmente adequadas). Ou seja, num cenário de cobertura nacional do pré-natal de cerca de $100 \%$, quase metade (43\%) das unidades de saúde pesquisadas não dispõe de infraestrutura adequada, revelando uma expressiva barreira de acesso a um pré-natal de qualidade.

Também tem sido apontado que a Região Norte, quando avaliada, tem tido a pior adequação para o pré-natal 8,10. Resultados similares foram encontrados na avaliação aqui empreendida, em que $51 \%$ das USF pesquisadas têm condição de funcionamento inadequada. Embora menores, tais percentuais são próximos aos achados para o Centro-oeste (48\%), Nordeste (44\%) e Sudeste (43\%), indicando que o país precisa ampliar seus investimentos em atenção básica, a fim de garantir condições adequadas à atenção pré-natal. São resultados que também evidenciam uma relação direta entre maior adequação da infraestrutura e regiões com renda e IDH-M mais elevados.

Publicação recente 2 que utilizou os dados da avaliação externa como fonte, contudo investigando as mulheres entrevistadas no PMAQ-AB, também concluiu que $75 \%$ das gestantes tiveram um prénatal inadequado e que a pior qualidade da atenção foi achada nas regiões Norte e Centro-oeste.

Por outro lado, não se demonstrou uma relação direta entre maior adequação de infraestrutura para o pré-natal e maiores índices de cobertura populacional pela atenção básica. A Região Sudeste, que teve taxa de inadequação de $43 \%$, possui uma das menores coberturas populacionais encontradas no estudo (70\%), ao passo que no Nordeste, que tem um percentual de adequação similar (44\%), tem a maior cobertura populacional pela rede básica no país (84\%). Já o Norte tem baixo desempenho em ambos os itens, e a Região Centro-oeste tem um perfil singular que conjuga baixa disponibilidade de infraestrutura, com baixa cobertura de atenção básica, em presença de renda domiciliar e IDH-M mais elevados do grupo.

São achados que sugerem interveniência de dimensões intersetoriais, cuja origem e repercussões não podem ser explicadas exclusivamente por características inerentes ao setor saúde ou ao pré-natal. Variáveis como a alta concentração populacional em municípios polo e maior acesso a serviços privados de saúde são apontados como razões para coberturas de atenção básica mais baixas, em regiões mais favorecidas economicamente, como o Sudeste 22. Em contraponto, o Norte e o Nordeste, onde os níveis de pobreza são os mais elevados do país, a população tem maior dependência da rede pública, explicando em parte as coberturas mais elevadas achadas no estudo 22 .

Estudos recentes de abrangência nacional 2,8,23 ou local 1,4 informam que, por mais que a cobertura do pré-natal esteja universalizada no país, as condições físicas de realização das atividades são precárias e o cuidado recebido nas unidades tem sido reconhecido como de baixa qualidade e baixa efetividade. Semelhante desempenho se mostra particularmente frágil quando os autores avaliam, no conjunto, as ações clínicas e laboratoriais necessárias à realização de uma atenção pré-natal adequada 4,10,24.

Nos resultados obtidos no banco do PMAQ-AB, observa-se que somente 1\% das ações clínicas ofertadas pelo pré-natal pode ser considerado adequado e 36\% parcialmente adequados para o conjunto do país. Somente quatro equipes informaram - e comprovaram - realizar todas as ações previstas no manual técnico do pré-natal. São resultados mais negativos que os relatados por Tomasi et al. 2 que encontraram, pelas entrevistas com as usuárias no âmbito da avaliação externa, 15\% de atenção pré-natal adequada. Tais diferenças se devem tanto à diversidade de fontes utilizadas (registros de ações de atenção pré-natal nas unidades de saúde versus depoimentos de usuárias) quanto ao modo de agregar os itens que formaram o indicador sintético que mediu a qualidade do pré-natal na publicação dos referidos autores e os aqui utilizados, que tomaram o PHPN como base para avaliar a oferta de ações clínicas.

Para a subdimensão cuidado, houve $40 \%$ de respostas que indicam uma qualidade do cuidado (compreendendo a oferta de ações clínicas e laboratoriais) parcialmente adequado para a Região Norte. É um achado não desprezível quando se relembra que a Região Norte obteve desempenho muito insatisfatório em outras dimensões do pré-natal. Vale dizer, ainda que o Norte tenha as piores condições de oferta de infraestrutura, alcançou, comparativamente a outras regiões, um nível mais adequado de qualidade do cuidado, aproximando-se da Região Sul (41\%), que alcançou desempenhos mais adequados em avaliações anteriores 7,8,10. Ainda assim, não se deve perder de vista que esses achados significam que cerca de $60 \%$ das grávidas não vêm recebendo um padrão mínimo de cuidados.

Foi encontrado apenas um estudo que analisou a gestão do cuidado na atenção básica 25 , mas não trata do pré-natal. Evidenciou-se nele uma baixa capacidade de gestão voltada a garantir o acesso 
às unidades de atenção básica. Achados deste estudo não evidenciaram diferenças expressivas no conjunto de regiões do país, somente percentuais ligeiramente mais elevados no Sudeste (30\% de adequação e $21 \%$ parcialmente adequado).

As ações gerenciais para garantir a qualidade do cuidado padecem de fragilidade ainda maior, evidenciando-se baixos percentuais de sucesso no apoio às atividades fim, já que foram encontrados percentuais ainda mais baixos de adequação plena nessa subdimensão. Ao rever as variáveis descritas na Tabela 1, observar-se-á que tais ações seriam justamente as que possibilitariam o registro, monitoramento e avaliação do pré-natal em cada USF, conforme as normas nacionais que orientam a realização do pré-natal. Os baixos percentuais aferidos podem responder, em grande medida, pelos resultados insatisfatórios obtidos na avaliação da qualidade do cuidado para todas as regiões.

O conjunto de dados relativos à avaliação do acesso e qualidade do pré-natal para os estados da Região Norte também não contou com apoio de publicações que pudessem enriquecer sua análise. $\mathrm{O}$ Estado de Roraima obteve melhor adequação da atenção pré-natal tanto no acesso (disponibilidade de infraestrutura das USF), quanto na qualidade do cuidado (ações clínicas). Em contrapartida, os estados do Amazonas e do Pará tiveram as piores adequações nessas mesmas subdimensões. Esses resultados corroboram os já descritos para as macrorregiões brasileiras, onde o perfil socioeconômico de cada estado possuía relação estreita com a adequação da atenção pré-natal.

Em relação às ações gerenciais, tanto para o acesso, quanto para a qualidade do cuidado, foram encontrados cenários semelhantes aos descritos para as cinco regiões geopolíticas: inadequação generalizada. Esses resultados revelam a importância e o impacto das ações de gerenciamento na avaliação global da atenção pré-natal, cujo desempenho inadequado pode contribuir para os piores resultados encontrados para a Região Norte em comparação às demais macrorregiões brasileiras.

Em estudo avaliativo anterior 7, foi demonstrado que os estados da Região Norte possuíam baixas adequações da atenção pré-natal comparativamente às demais regiões, com destaque para o Amazonas que não dispunha sequer de dados das gestantes cadastrados no sistema de informação à época. Avanços ocorreram, no entanto o Amazonas continua com problemas, tendo obtido a pior adequação do pré-natal, em todas as subdimensões avaliadas, seguido pelo Pará. Esses dois estados também têm as piores condições de renda, IDH-M e cobertura populacional pela atenção básica na Região Norte.

No conjunto, não se encontrou coexistência entre níveis elevados de adequação da atenção prénatal e melhores indicadores de cobertura populacional pela atenção básica nos estados do Norte, tal como verificado também para as macrorregiões geográficas do país. O mesmo ocorreu ao se cruzar o desempenho da atenção pré-natal com as taxas de mortalidade neonatal de cada estado. A redução das taxas de mortalidade neonatal expressa melhoria da qualidade da atenção pré-natal, porém tais taxas são influenciadas por ampla gama de fatores que abrangem não só a qualidade do cuidado pré-natal, mas outras variáveis fora do escopo deste estudo, como as condições de acesso à maternidade e a qualidade da assistência ao parto ${ }^{26}$, cuja interveniência pode influenciar nos achados desta pesquisa.

Os limites do estudo abrangem aspectos como a adesão voluntária das equipes, que poderia gerar algum comprometimento da validade da pesquisa pela falta de aleatoriedade da amostra. Entretanto, uma amostra que alcançou quase $90 \%$ das equipes atuantes em todo o território brasileiro, à época da coleta dos dados, diminui esse viés de seleção. Outro limite se refere ao tipo de estudo, pois a avaliação normativa não propicia a compreensão das causas e efeitos na relação entre a intervenção avaliada e seus resultados 27,28. Ademais, os dados analisados concernem apenas às equipes da ESF e não abordam outros tipos de conformações de equipes que existem na atenção básica. Por fim, as questões disponíveis no banco de dados do PMAQ-AB não continham todos os itens habitualmente utilizados na literatura para avaliar o pré-natal, impossibilitando a aferição mediante índices tradicionais como Kessner e Kotelchuck.

\section{Considerações finais}

O aprimoramento da qualidade do pré-natal e a ampliação de seu acesso no âmbito da ESF são iniciativas relevantes, pois sua adequada estruturação e oferta têm resultados positivos na redução da morbimortalidade materna e infantil. 
Os resultados aqui analisados revelaram importantes dificuldades organizacionais tanto no acesso, quanto na qualidade do cuidado ofertado pelas EqSF no país inteiro, além de uma evidente inadequação das ações de gestão voltadas ao aprimoramento do cuidado pré-natal. As desigualdades regionais, sobretudo no acesso, foram expressivas e ratificam a necessidade do fortalecimento de políticas públicas que objetive diminuí-las e aprimorar a qualidade dos serviços oferecidos à população.

O acesso e a qualidade do pré-natal também estão ligados às condições de vida da população de cada macrorregião e/ou estado estudado. Ainda que os investimentos no setor saúde não possam reverter indicadores sociais negativos, a garantia do acesso universal e equânime às gestantes pode contribuir para reduzir desigualdades, sobretudo para aquelas de baixa renda, que mais necessitam dos serviços públicos do pré-natal.

A originalidade da abordagem, ao abranger dimensões gerenciais anteriormente não exploradas pela literatura, permitiu apreender lacunas na dinâmica do pré-natal que - em grande medida podem explicar as limitações na qualidade do cuidado, globalmente apontadas na literatura.

\section{Colaboradores}

W. S. G. Guimarães contribuiu na concepção e desenvolvimento da pesquisa, aplicação de testes estatísticos e elaboração e revisão final do artigo. R. C. P. Parente contribuiu no desenvolvimento da pesquisa, elaboração dos testes estatísticos e revisão dos resultados da versão final do artigo. T. L. F. Guimarães contribuiu no desenvolvimento da pesquisa e revisão final do artigo. L. Garnelo contribuiu na concepção e desenvolvimento da pesquisa, elaboração e revisão final do artigo.

\section{Agradecimentos}

Aos professores do Instituto Leônidas e Maria Deane, Fundação Oswaldo Cruz (ILMD/Fiocruz) e da Universidade Federal do Amazonas (UFAM) vinculados ao Programa de Pós-graduação em Saúde, Sociedade e Endemias na Amazônia pelo apoio para a realização deste estudo.

\section{Referências}

1. Mendoza-Sassi RA, Cesar JA, Teixeira TP, Ravache C, Araújo GD, Silva TC. Diferenças no processo de atenção ao pré-natal entre unidades da Estratégia Saúde da Família e unidades tradicionais em um município da Região Sul do Brasil. Cad Saúde Pública 2011; 27:787-96.

2. Tomasi E, Fernandes PAA, Fisher T, Siqueira FCV, Silveira DS, Thumé E, et al. Qualidade da atenção pré-natal na rede básica de saúde do Brasil: indicadores e desigualdades sociais. Cad Saúde Pública 2017; 33:e00195815.

3. Departamento de Ações Programáticas Estratégicas, Secretaria de Atenção à Saúde, Ministério da Saúde. Pré-natal e puerpério: atenção qualificada e humanizada - manual técnico. Brasília: Ministério da Saúde; 2005.

4. Dias-da-Costa G, Cotta RMM, Reis JR, Siqueira-Batista R, Gomes AP, Franceschini SCC. Avaliação do cuidado à saúde da gestante no contexto do Programa Saúde da Família. Ciênc Saúde Coletiva 2009; 14:1347-57.

5. Piccini RX, Facchini LA, Tomasi E, Thumé E, Silveira DS, Siqueira FV, et al. Efetividade da atenção pré-natal e de puericultura em unidades básicas de saúde do Sul e do Nordeste do Brasil. Rev Bras Saúde Matern Infant 2007; 7:75-82.

6. Ministério da Saúde. Portaria no 1.654, de 19 de julho de 2011. Institui, no âmbito do Sistema Único de Saúde, o Programa Nacional de Melhoria do Acesso e da Qualidade da Atenção Básica (PMAQ-AB) e o Incentivo Financeiro do PMAQ-AB, denominado Componente de Qualidade do Piso de Atenção Básica Variável - PAB Variável. Diário Oficial da União 2011; 20 jul. 
7. Serruya SJ, Lago TG, Cecatti JG. Avaliação preliminar do Programa de Humanização no Pré-natal e Nascimento no Brasil. Rev Bras Ginecol Obstet 2004; 26:517-25.

8. Viellas EF, Domingues RMSM, Dias MAB, Gama SGN, Theme Filha MM, Costa JV, et al. Assistência pré-natal no Brasil. Cad Saúde Pública 2014; 30 Suppl 1:S85-100.

9. Costa AM, Guilhem D, Walter MIMT. Atendimento a gestantes no Sistema Único de Saúde. Rev Saúde Pública 2005; 39:768-74.

10. Domingues RMSM, Viellas EF, Dias MAB, Torres JA, Theme-Filha MM, Gama SGN, et al. Adequação da assistência pré-natal segundo as características maternas no Brasil. Rev Panam Salud Pública 2015; 37:140-7.

11. Neumann NA, Tanaka OY, Victora CG, Cesar JA. Qualidade e equidade da atenção ao prénatal e ao parto em Criciúma, Santa Catarina, Sul do Brasil. Rev Bras Epidemiol 2003; 6:30718.

12. Parada CMGL. Avaliação da assistência prénatal e puerperal desenvolvidas em região do interior do Estado de São Paulo em 2005. Rev Bras Saúde Matern Infant 2008; 8:113-24.

13. Departamento de Atenção Básica, Secretaria de Atenção à Saúde, Ministério da Saúde. Documento síntese para avaliação externa do Programa Nacional de Melhoria do Acesso e da Qualidade da Atenção Básica (PMAQ). Brasília: Ministério da Saúde; 2012.

14. Pinto HA, Sousa A, Florêncio AR. O Programa Nacional de Melhoria do Acesso e da Qualidade da Atenção Básica: reflexões sobre o seu desenho e processo de implantação. RECIIS (Online) 2012; 6(2). https://www.reciis.icict. fiocruz.br/index.php/reciis/article/view/492.

15. Travassos C, Martins M. Uma revisão sobre os conceitos de acesso e utilização de serviços de saúde. Cad Saúde Pública 2004; 20 Suppl 2:S190-8.

16. Champagne F, Brousselle A, Hartz Z, Contandriopoulos AP. A apreciação normativa. In: Brousselle A, Champagne F, Contandriopoulos AP, Hartz Z, organizadores. Avaliação: conceitos e métodos. Rio de Janeiro: Editora Fiocruz; 2011. p. 77-94.

17. Alves CKA, Natal S, Felisberto E, Samico I. Interpretação e análise das informações: o uso de matrizes, critérios, indicadores e padrões. In: Samico I, Felisberto E, Figueiró AC, Frias PG, organizadores. Avaliação em saúde: bases conceituais e operacionais. Rio de Janeiro: MedBook; 2010. p. 89-107.

18. Tomasi E, Oliveira TF, Fernandes PAA, Thumé E, Silveira DS, Siqueira FV, et al. Estrutura e processo de trabalho na prevenção do câncer de colo de útero na Atenção Básica à Saúde no Brasil: Programa de Melhoria do Acesso e da Qualidade - PMAQ. Rev Bras Saúde Matern Infant 2015; 15:171-80.
19. Silveira DS, Santos IS, Costa JSD. Atenção prénatal na rede básica: uma avaliação da estrutura e do processo. Cad Saúde Pública 2001; 17:131-9.

20. Moura BLA, Cunha RC, Fonseca ACF, Aquino R, Medina MG, Vilasbôas ALQ, et al. Atenção primária à saúde: estrutura das unidades como componente da atenção à saúde. Rev Bras Saúde Matern Infant 2010; 10 Suppl 1:S69-81.

21. Giovanella L, Bousquat A, Fausto MCR, Fusaro ER, Mendonça MHM, Gagno J. Tipologia das unidades básicas de saúde brasileiras. Brasília: Região e Redes; 2015. (Nota Técnica, 5/2015).

22. Duarte CMR, Pedroso MM, Bellido JG, Moreira RS, Viacava F. Regionalização e desenvolvimento humano: uma proposta de tipologia de Regiões de Saúde no Brasil. Cad Saúde Pública 2015; 31:1163-74.

23. Victora CG, Aquino EML, do Carmo Leal M, Monteiro CA, Barros FC, Szwarcwald CL. Maternal and child health in Brazil: progress and challenges. Lancet 2011; 377:1863-76.

24. Martinelli KG, Santos Neto ET, Gama SGN, Oliveira AE. Adequação do processo da assistência pré-natal segundo os critérios do Programa de Humanização do Pré-natal e Nascimento e Rede Cegonha. Rev Bras Ginecol Obstet 2014; 36:56-64.

25. Sarti TD, Campos CEA, Zandonade E, Ruschi GEC, Maciel ELN. Avaliação das ações de planejamento em saúde empreendidas por equipes de saúde da família. Cad Saúde Pública 2012; 28:537-48.

26. Lansky S, Friche AAL, Silva AAM, Campos D, Bittencourt SDA, Carvalho ML, et al. Pesquisa Nascer no Brasil: perfil da mortalidade neonatal e avaliação da assistência à gestante e ao recém-nascido. Cad Saúde Pública 2014; 30 Suppl 1:S192-207.

27. Contandriopoulos AP. Avaliando a institucionalização da avaliação. Ciênc Saúde Coletiva 2006; 11:705-11.

28. Vieira-da-Silva LM. Conceitos, abordagens e estratégias para a avaliação em saúde. In: Hartz ZMA, Vieira-da-Silva LM, organizadores. Avaliação em saúde: dos modelos teóricos à prática na avaliação de programas e sistemas de saúde. Salvador: EDUFBA/Rio de Janeiro: Editora Fiocruz; 2005. p. 15-40. 


\section{Abstract}

This study focuses on access to prenatal care and quality of care in the Family Health Strategy in Brazil as a whole and in the North region, through evaluation of infrastructure characteristics in the health units, management, and supply of care provided by the teams, from the perspective of regional and state inequalities. A cross-sectional evaluative and normative study was performed, drawing on the external evaluation component of the second round of the Program for Improvement of Access and Quality of Primary Care, in 2013-2014. The results revealed the inadequacy of the primary healthcare network's infrastructure for prenatal care, low adequacy of clinical actions for quality of care, and the teams' low management capacity to guarantee access and quality of care. In the distribution according to geopolitical regions, the findings pertaining to the units' infrastructure indicate a direct relationship between the infrastructure's adequacy and social contexts with higher municipal human development indices and income. For the clinical actions in patient care, the teams in all the regions scored low on adequacy, with slightly better results in the North and South regions of the country. There were important differences between the states of the North, and the states with higher mean income and human development scored higher on adequacy. The results indicate important organizational difficulties in both access and quality of care provided by the health teams, in addition to visible insufficiency in management activities aimed to improve access and quality of prenatal care.

Primary Health Care; Prenatal Care; Health Evaluation; Health Inequalities

\section{Resumen}

Se trata de un estudio sobre el acceso y calidad del cuidado prenatal en la Estrategia Salud de la Familia en Brasil y en la región Norte, mediante la evaluación de aspectos de infraestructura en las unidades de salud, de la gestión, así como de los servicios de cuidado prestados por los equipos; todo ello desde el prisma de las desigualdades regionales y estatales. Se realizó un estudio transversal, evaluativo de tipo normativo, procedente del componente de evaluación externa del segundo ciclo del Programa de Mejora del Acceso y de la Calidad de la Atención Básica, realizado entre 2013-2014. Los resultados evidencian la inadecuación de la infraestructura de la red de atención básica que realiza el seguimiento prenatal; baja adecuación de acciones clínicas para la calidad del cuidado y baja capacidad de gestión de los equipos para garantizar el acceso y calidad a los cuidados establecidos. En la distribución por regiones geopoliticas, los hallazgos relativos a la infraestructura de las unidades apuntan una relación directa entre adecuación de la infraestructura y contextos sociales con un Índice de Desarrollo Humano Municipal y renta más elevados. Para las acciones clínicas del cuidado, los equipos de todas las regiones obtuvieron índices bajos de adecuación, habiéndose observado resultados discretamente más elevados en las regiones Norte y Sur. Hubo diferencias expresivas entre los estados de la región Norte, obteniendo una mejor adecuación las unidades federadas con mejores condiciones de renta y de desarrollo humano. Los resultados indican importantes dificultades organizativas tanto en el acceso, como respecto a la calidad del cuidado ofertado por los equipos de salud, además de una evidente insuficiencia de las acciones de gestión, dirigidas a la optimización del acceso y calidad del cuidado prenatal.

Atención Primaria de Salud; Atención Prenatal; Evaluación en Salud; Desigualdades en la Salud

Recebido em 27/Jun/2017

Versão final reapresentada em 19/Set/2017

Aprovado em 24/Out/2017 